\title{
Proximal Disease Extension in Patients With Limited Ulcerative Colitis: A Danish Population- based Inception Cohort
}

\author{
Johan Burisch, ${ }^{a}$ Ryan Ungaro, ${ }^{b}$ Ida Vind, ${ }^{a}$ Michelle V. Prosberg, ${ }^{a}$ \\ Flemming Bendtsen, ${ }^{\mathrm{a}}$ Jean-Frederic Colombel, ${ }^{\mathrm{b}}$ \\ Marianne K. Vester-Andersen ${ }^{\mathrm{a}, \mathrm{c}}$
}

aGastrounit, Medical Division, Copenhagen University Hospital Hvidovre, Hvidovre, Denmark 'busan and Leonard Feinstein IBD Clinical Center, Icahn School of Medicine at Mount Sinai, New York, USA 'Department of Internal Medicine, Zealand University Hospital, Køge, Denmark

Corresponding author: Johan Burisch, MD, PhD, Gastrounit, Medical Division, Copenhagen University Hospital, Hvidovre, Kettegård Allé 30, 2750 Hvidovre, Denmark. Email: johan.burisch@regionh.dk

\begin{abstract}
Background and Aims: Disease extent in ulcerative colitis [UC] is dynamic and can progress over time. Little is known about risk factors for UC extension in the era of biologics. We investigated the risk of UC extension and subsequent risk of surgery in a Danish population-based cohort.

Methods: All incident UC cases in a strictly defined Copenhagen area between 2003 and 2004 were followed prospectively through 2011. Disease extension was defined as patients with limited UC [E1 or E2] at diagnosis having progressed from the initial extent by colonoscopy or surgery to E2 or extensive colitis [E3]. Associations between progression or colectomy and multiple covariates were analysed by Cox regression analysis.

Results: Of 300 UC patients, 220 [73\%] had E1 or E2 at diagnosis. During follow-up, 50 [23\%] patients with E1/E2 progressed to E3, and 22 [10\%] with E1 progressed to E2. Disease extent at diagnosis was the sole predictor of extension to E3. A total of 18 [8\%] patients with E1/E2 at diagnosis had a colectomy. Progression from E1/E2 to E3, female gender and a history of smoking were risk factors for colectomy.

Conclusion: After 7 years of follow-up, 33\% of patients with limited UC experienced disease extension. Only extent at diagnosis was a clinical predictor for disease extension. The risk of colectomy was increased in former smokers and patients who progressed to extensive colitis. This highlights the need to prevent disease progression in patients with limited UC, and to identify new histological or molecular markers that might help stratify risks for disease progression.
\end{abstract}

Key Words: ulcerative colitis; colectomy; disease extent; inception cohort

\section{Introduction}

Ulcerative colitis [UC] causes chronic mucosal inflammation involving the rectum that can extend proximally to involve the entire colon. ${ }^{1}$ Disease extent in UC is classified as proctitis [E1], left-sided [E2] or extensive colitis [E3]. ${ }^{2}$ The extent to which the colon is involved has a major impact on treatment and long-term disease prognosis. UC patients with greater disease extent are more likely to require more aggressive therapy and with an increased need for steroids. ${ }^{3}$ Extensive colitis has been associated with higher rates of hospitalizations, surgeries and possibly mortality. ${ }^{4-8}$ Furthermore, 
patients with left-sided and extensive colitis are at increased risk of developing colorectal cancer compared to those with proctitis. ${ }^{9}$

UC is a dynamic disease that can progress to involve increasing segments of the colon over time. ${ }^{10}$ In population-based studies, around two-thirds of UC patients have limited disease [E1 or E2] at diagnosis but nearly $30 \%$ will have proximal disease extension within 10 years. ${ }^{11,12}$ Patients who experience disease extension associated with a UC flare-up are more likely to need immunosuppressants and surgery than those who do not. ${ }^{13,14}$ It is therefore important to identify UC patients who are at increased risk for extension in order to monitor them more closely and introduce effective therapies earlier. Most prior studies have principally investigated patients diagnosed with UC in the pre-biologic era and the means of assessing disease extent have ranged from barium enema and sigmoidoscopy to colonoscopy. The aim of this study was to identify risk factors for disease extension and subsequent colectomy in a Danish populationbased, prospective cohort of UC patients diagnosed in the era of biologics with disease extent, as assessed by colonoscopy.

\section{Material and Methods}

\subsection{Patient population}

All incident patients diagnosed with UC, Crohn's disease [CD] or irritable bowel disease Unclassified [IBDU] between January 1, 2003 and December 31, 2004 in a clearly defined Copenhagen area from a reference population of 1211634 residents [ $23 \%$ of the total population of Denmark] were prospectively included in a population-based inception cohort. ${ }^{15}$ Registration was carried out by 28 specialist practitioners [gastroenterologists and surgeons] in ten departments of gastroenterology and internal medicine, including two paediatric units, covering all eight hospitals in the region. All specialist practitioners from this same area were contacted and asked to list their newly diagnosed IBD patients. Fifteen did not have any IBD patients, three practitioners did not wish to participate and the remaining 28 provided complete lists of all newly diagnosed IBD patients who had not already been referred to one of the regional hospitals. At the end of the study period, discharge and outpatient clinic registries were searched to ensure completeness of the database. ${ }^{15}$

Patients were followed from the date of diagnosis to December 31,2011 , ensuring a follow-up period of at least 7 years. ${ }^{16}$ Clinical data including demographics, medical therapy, surgery and hospitalization rates, investigations, and changes in disease classification were registered throughout the follow-up period. All cases were reevaluated and patients who were found not to fulfil the diagnostic criteria for UC [including patients with IBDU] or who were not incident were excluded from the follow-up analyses. Details regarding the re-evaluation have been described previously. ${ }^{16}$ Patients who died or emigrated were excluded from the study at the time of death or on the date of emigration.

According to Danish regulations, all new prescriptions or changes in medication are to be documented in the individual's patient record. Complete data capture regarding surgical procedures for the entire study group was ensured by linking the patients' unique ten-digit personal identification number to the National Patient Registry, ${ }^{17}$ which contains information on diagnosis codes and surgical procedures. A comparison with the surgical procedure registry was performed by cross-matching codes for resection and colectomy [KJFB00 - 97, KJFHH00 - 96] with codes for IBD [K50.0 - K51.9].

\subsection{Diagnosis and definitions}

The diagnosis of UC was based on the Copenhagen Diagnostic Criteria. ${ }^{18-20}$ The extent of disease in UC was defined according to the Montreal classification as E1: proctitis [proximal extent to the sigmoid colon], E2: left-sided [to the splenic flexure] or E3: extensive disease [beyond the splenic flexure]. ${ }^{2}$ Disease extension was defined as a proximal progression from the initial extent at diagnosis, as defined by endoscopy. In cases where no investigative procedures were performed before surgery owing to acute symptoms, macroscopic description of the surgical specimen was used to describe the disease extent. Smoking behaviour was recorded at inclusion. Smoking was defined as daily consumption of tobacco for at least 6 months prior and was stratified as 'ongoing smoker', 'former smoker' or 'never smoker'. Diagnostic delay was defined as the number of days from the onset of symptoms until diagnosis.

Treatment was grouped according to five levels by ascending therapeutic potency: 5-aminosalicylates [5-ASA] [oral and/or topical 5-ASA treatment \pm topical steroids], corticosteroids [CS] [oral steroids \pm 5 -ASA or topical steroids], immunosuppressants [azathioprine or 6-mercaptopurine \pm steroids], biologics [infliximab or adalimumab in combination with any of the above] and surgery [major abdominal surgery due to IBD regardless of medical treatment prior to surgery].

\subsection{Treatment policy}

The treatment regimen for IBD in Copenhagen during the followup period was based on Danish IBD guidelines, which agree with European [European Crohn's and Colitis Organization], British [British Society of Gastroenterology] and American [American Gastroenterological Association] guidelines. In UC, 5-ASA was used for the treatment of non-severe flare-ups, maintenance and first-line prophylaxis [topical and/or oral]. This drug was supplemented with systemic CS in severe flare-ups and, if this did control the disease, with immunosuppressants [azathioprine $2.0-2.5 \mathrm{mg} / \mathrm{kg}, 6$-mercaptopurine $1.0-1.5 \mathrm{mg} / \mathrm{kg}$ ] as second-line prophylaxis. The treatment of UC with infliximab was approved in 2006. Adalimumab was approved for UC treatment in 2011 in Denmark. The Regional Ethics Committee approved this study [H-1-2011-088], and permission was obtained from the Danish Data Registry [01769 HVH-2012-027].

\subsection{Statistical analysis}

The statistical analyses were performed with SAS [version 9.3] statistical software [SAS Institute]. The data are given as numbers and percentages or medians and ranges. All medications taken before diagnosis were disregarded. Associations between progression of disease extent or colectomy, along with multiple covariates [age at diagnosis, gender, initial disease extent, type of medical treatment, diagnostic delay and smoking status at diagnosis], were analysed by Cox regression analyses using the proportional hazard assumption. Age at diagnosis was included in the analysis as both a continuous and a categorical variable using the age groups $<18,18-40,40-60$ and $>60$ years. The risk of colectomy was assessed in patients experiencing disease extension as well as in all incident UC patients. A $p$-value of $<0.05$ was considered statistically significant.

\section{Results}

A total of 300 patients fulfilled the diagnostic criteria for UC and were included in the study. The median follow-up time was 7.5 years (interquartile range [IQR] 5.6-8.1). Patient characteristics are shown in Table 1.

\subsection{Change in disease extent during follow-up}

Disease extent at diagnosis and maximal extent during follow-up of the patients is shown in Table 2. At the time of diagnosis, 220 [73\%] 
Table 1. Patient characteristics of incident patients with ulcerative colitis.

\begin{tabular}{lc}
\hline & Ulcerative colitis \\
\hline No. of patients, $n$ & 300 \\
Age [years] at diagnosis, median [range] & $37.3[1.5-93.5]$ \\
Years of follow-up, median [range] & $7.5[0.0-9.0]$ \\
Gender, $n$ [\%] & \\
$\quad$ Male & $151[50.3]$ \\
Female & $149[49.7]$ \\
Smoking habits at diagnosis, $n[\%]$ & \\
Never smoked & $137[45.7]$ \\
Current smoker & $45[15.0]$ \\
Former smoker & $72[24.0]$ \\
Disease extent at diagnosis, $n[\%]$ & \\
E1 & $93[31.0]$ \\
E2 & $127[42.3]$ \\
E3 & $80[26.7]$ \\
Medical treatment during follow-up, $n[\%]$ & \\
5-Aminosalocylates & $276[92.0]$ \\
Corticosteroids & $220[73.3]$ \\
Immunomodulators & $78[26.0]$ \\
Biological therapy & $18[6.0]$ \\
\hline
\end{tabular}

Table 2. Disease extent in UC at diagnosis and follow-up.

\begin{tabular}{llccc}
\hline At diagnosis & \multicolumn{2}{l}{ At follow-up } & \multirow{2}{*}{ Total [diagnosis] } \\
\cline { 2 - 4 } & E1 & E2 & E3 & \\
\hline E1 & $58[26 \%]$ & $22[10 \%]$ & $13[6 \%]$ & $93[42 \%]$ \\
E2 & - & $90[41 \%]$ & $37[17 \%]$ & $127[58 \%]$ \\
Total [follow-up] & $58[26 \%]$ & $112[51 \%]$ & $50[23 \%]$ & $220[100 \%]$ \\
\hline
\end{tabular}

E1, proctitis; E2, left-sided; E3, extensive disease.

Values shown are number [percentage of total]; bold type indicates patients without change in disease extent during follow-up.

patients had limited UC [E1 or E2]. During the follow-up period and of the patients diagnosed with E1, 22 [24\%] progressed to E2 and 13 [14\%] progressed to E3. Of patients diagnosed with E2, 37 [29\%] progressed to E3. Overall, 50 [23\%] patients with E1/E2 progressed to E3, of whom 41 [19\%] did so within the first 5 years. The median time for any progression of disease extent was 12 months [range: 0-106]; more specifically it was 13 months [range: 1-106] for progression from E1 to E2, 15 months [range: 0-80] for progression from E1 to E3, and 8 months [range: 0-91] for progression from E2 to E3.

Cox regression analysis found that disease extent at diagnosis was the sole significant predictor of extension to E3, with a higher risk in E2 than in E1 patients (hazard ratio [HR] 2.2, 95\% confidence interval [CI] 1.2-4.2) [Figure 1]. No significant predictors were found for extension from E1 to E2.

\subsection{Risk of colectomy}

During the follow-up period, a total of 34 [11\%] patients had a colectomy. Specifically, colectomy was performed in seven [8\%] patients with E1, 12 [9\%] patients with E2 and 15 [19\%] with E3 at diagnosis. Median time from diagnosis to colectomy in all patients was 7 months [range: 0-85]; 10 months [range: 4-80] in E1 patients, 17 months [range: $0-85$ ] in E2 patients and 2 months [range: 0-81] in E3 patients. Overall, only disease extent at diagnosis [E3 vs E1: HR 2.9, 95\% CI 1.1-7.5; E2 vs E1: HR 1.2, $95 \%$
CI 0.5-3.0] as well as a diagnostic delay of more than 6 months [HR 2.5, 95\% CI 1.1-5.7] were found to be associated with the risk of colectomy. Before colectomy, four [20\%] patients with E3 at diagnosis had received biological therapy, fove [33\%] had received immunomodulators, while five [33\%] had received system corticosteroids as the highest treatment step. Two [13\%] were colectomized immediatly following diagnosis.

The analysis of risk factors associated with colectomy in patients with limited UC is shown in Table 3. Progression from E1/E2 to E3 and the need for systemic corticosteroids within the first year of diagnosis were significant risk factors for colectomy. Among patients with limited UC requiring colectomy, all 19 patients [100\%] had received 5-ASA and systemic corticosteroids while only eight [42\%] had received immunosuppressants and four [21\%] had received biological therapy prior to surgery.

When analysing the risk of colectomy in patients with either E3 at diagnosis or with limited UC at diagnosis who progressed to E3 during follow-up, Cox regression analysis found no difference in the colectomy risk between these two groups of patients [E3 vs. E1/E2 extenders: HR $0.6,95 \%$ CI 0.3-1.2]. A diagnostic delay of less than 6 months increased the risk of colectomy in this analysis [HR 2.3, $95 \%$ CI 1.01-5.3]. The risk of colectomy was higher in patients with E3 or E1/E2 extenders compared to E1/E2 non-extenders [Figure 2].

\section{Discussion}

We have shown that in an unselected, population-based, inception cohort of mild-to-severe UC cases in Copenhagen, Denmark, one in three UC patients experienced some degree of disease extension [E1 to E2/E3 or E2 to E3] after 7 years of follow-up. One in four patients with limited UC experienced disease extension to extensive colitis. Only disease extent at diagnosis was identified as a clinical predictor for disease extension. Progressing to extensive colitis resulted in a higher risk for colectomy, and the risk of colectomy in those who progressed in disease extent was comparable to the risk of patients with extensive colitis at baseline.

Previous estimates in the literature for rates of disease extension vary widely due to differences in study design, treatment strategies and ascertainment of extent. Only a few population-based cohorts have reported on disease extension while most data originate from referral centre studies, which carry the risk of selection bias. In a Danish inception cohort from 1962 to $1987,27 \%$ of patients with proctosigmoiditis experienced further progression of their disease after 5 years. ${ }^{21}$ In a Norwegian inception cohort from 1990 to 1994 , $14 \%$ of patients with limited UC had progressed to extensive colitis after 5 years, and the rate increased to $21 \%$ after 10 years; overall, $40 \%$ of UC patients experienced progression after 10 years. ${ }^{22}$ The rate of extension in the present cohort was found to be comparable with, if slightly higher than, these figures despite the similar study design and the extent being assessed with endoscopy or during surgery. A more recent Hungarian inception cohort study found that $13 \%$ of patients with limited UC progressed to extensive colitis after 5 years. ${ }^{23}$ Finally, a recent meta-analysis of 28 studies found an overall pooled extension rate of $27 \%$, which is in line with our findings. ${ }^{11}$

In the current study, the only predictor for UC extension was having E2 extent at baseline compared to E1. This finding suggests that clinicians need to follow E2 patients more closely and should be aware of their higher risk for progression. Other risk factors that have been proposed in the past for disease extension are patients diagnosed at a younger age,,$^{22,24,25}$ family history of UC and a personal history of primary sclerosing cholangitis..$^{24,26}$ In the current 
Table 3. Risk factors associated with colectomy in ulcerative colitis patients with proctitis or left-sided colitis.

\begin{tabular}{lc}
\hline & $\begin{array}{l}\text { Hazard ratio } \\
{[95 \% \text { confidence interval] }}\end{array}$ \\
\hline Ex-smoker at diagnosis & $2.51[0.89-7.09]$ \\
Younger age at diagnosis & $0.98[0.95-1.02]$ \\
Gender [female vs male] & $2.12[0.76-5.93]$ \\
Corticosteroids during first year & $6.94[1.79-26.87]^{*}$ \\
Extension from E1/ E2 to E3 & $21.93[4.82-99.72]^{*}$ \\
Diagnostic delay <6 months & $2.02[0.68-6.01]$ \\
Disease extent at diagnosis & $0.35[0.12-1.01]$ \\
$\quad$ E2 vs E1 & \\
\hline
\end{tabular}

$* P<0.05$.

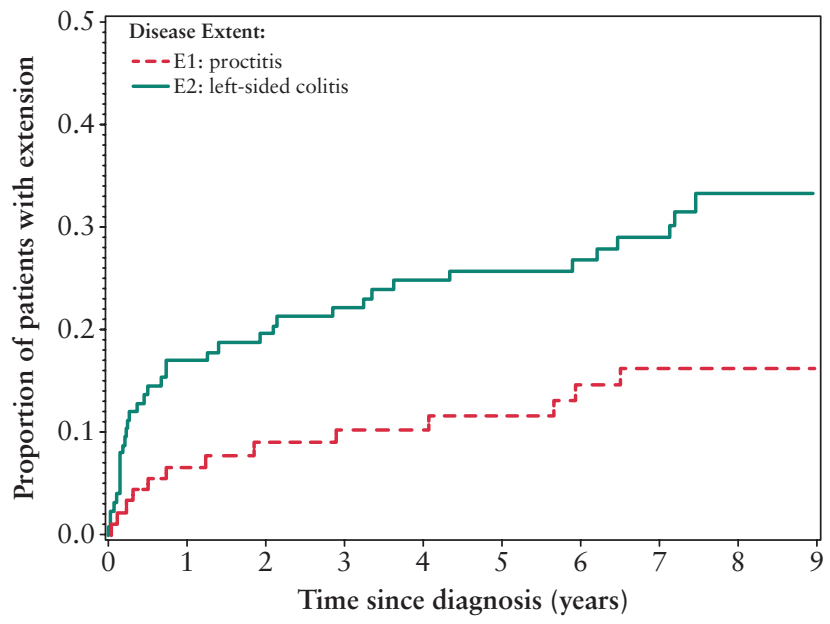

Figure 1. Proportion of patients with proctitis or left-sided colitis at diagnosis progressing to extensive colitis in a Danish population-based inception cohort.

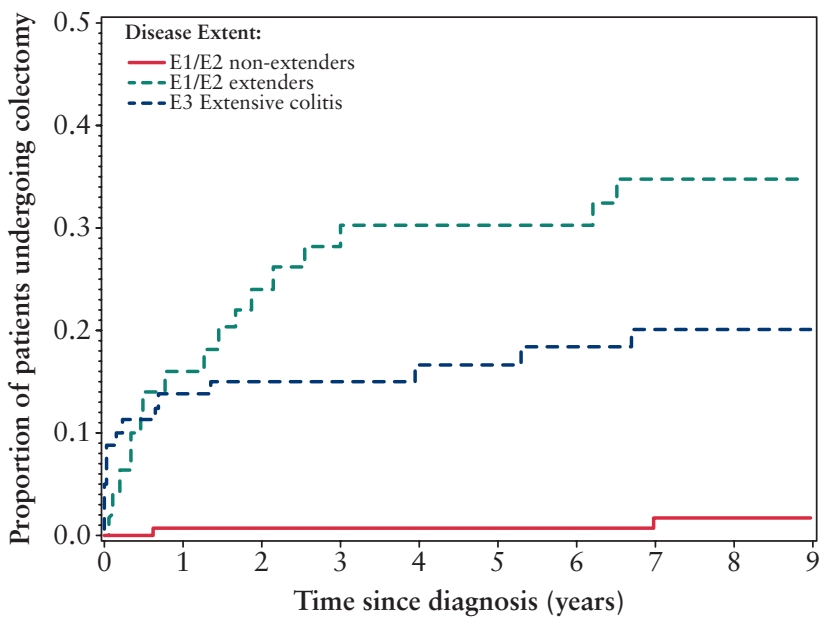

Figure 2. Proportion of patients with ulcerative colitis undergoing colectomy in a Danish population-based inception cohort.

study, there was a trend toward increased risk of progression in patients diagnosed at a younger age but this was not statistically significant. Patients with disease extension appear to be more likely to have had refractory or chronically active UC. ${ }^{27,28}$ Finally, smoking may have a protective effect against proximal disease extension. ${ }^{27,29}$ However, risk factors have been largely inconsistent across studies and better predictors of disease progression are needed.

We found that UC disease extension was associated with a significantly increased risk of colectomy. Few prior studies have examined the relationship between extension and outcomes. ${ }^{22,24,26,30}$ For example, progression from limited to extensive UC was preceded by a flare-up in the majority of patients in a single-centre retrospective study. ${ }^{30}$ In the population-based IBSEN cohort, there was a trend toward higher cumulative rates of colectomy in extenders as compared to patients with extensive disease at baseline. ${ }^{22}$ One prior study analysing an earlier period [1996-2006] specifically investigated the relationship between extension and outcomes. ${ }^{24}$ In the present study, in which disease extent was assessed by endoscopy and/or transabdominal ultrasound, UC extension resulted in poorer outcomes. Patients with progression from limited to extensive colitis were more likely to be steroid-refractory, need thiopurines or cyclosporine, require hospitalization and undergo colectomy, as compared to those with stable limited disease. Interestingly, in comparison to flare-ups in patients who had experienced extensive disease since diagnosis, flare-ups accompanied by extension of UC from limited to extensive disease had poorer outcomes, including the need for hospitalizations and surgery. Similarly, we found that disease extension significantly increased the chance of colectomy when compared to stable limited disease. Furthermore, in our cohort the risk of colectomy in extenders was higher, but not significantly so, than colectomy rates in patients with extensive disease since diagnosis. These findings suggest that patients with disease extension have a prognosis that is at least similar to, and potentially worse than, patients with long-standing extensive UC.

The major strength of the present cohort study is the populationbased design in a setting with free access to public healthcare in a well-defined area constituting a large segment of the total population. By including unselected incident IBD patients, this cohort represents the whole spectrum of disease severity. Patient records from the entire follow-up period were evaluated, diagnoses were reassessed according to strict diagnostic criteria and only patients fulfilling these criteria were included in the follow-up analysis. In addition, disease extent was defined by colonoscopy and/or surgery and categorized according to the most current Montreal classification. The use of incident cases and re-evaluation of diagnosis at follow-up limited any bias arising from misdiagnosis. The personalized ten-digit registration number unique to Danish patients enabled us to ensure complete follow-up regarding medical treatment and surgery until the end of the study. Limitations of the study include the fact that although the cohort was followed prospectively, data collection was not performed using consecutive case record forms at every outpatient visit. This may have led to greater variability in the interpretation of the results of diagnostic procedures. Furthermore, findings from this study might not be fully transferable to other geographical regions due to the quality of, and free access to, the Danish healthcare system.

Unanswered questions remain despite the current literature on UC extension. First, greater clarity on which clinical risk factors are truly associated with UC extension is needed. Second, it is unknown if any treatments or interventions decrease the risk of disease extension. Third, because there are currently no clear clinical predictors of UC extension, future research should investigate histological or molecular markers that could predict progression from E1/E2 to E3.

In conclusion, this 7-year follow-up of an inception cohort demonstrates that UC is a dynamic disease and that a significant proportion 
of patients progress in disease extent over time. Progressing from limited UC to extensive colitis increased the risk of colectomy significantly to a level comparable to those diagnosed with extensive colitis. Our findings highlight the need of preventing progression in patients with limited UC, as well as the need to identify markers [clinical, histological and molecular] that might stratify patients based on their risk of disease progression.

\section{Funding}

Unrestricted grant support was received from the Kirsten and Freddy Johansens Fond. RU is supported by a Career Development Award from the Crohn's and Colitis Foundation of America.

\section{Conflict of Interest}

JB: lecture fees from AbbVie, Takeda and MSD; consulting fees from AbbVie, Celgene, and Janssen. RU: consulting fees from Pfizer. IV: none. MVP: none. FB: none. JFC: consultant, advisory board member or speaker for AbbVie, Amgen, Boehringer-Ingelheim, Celgene Corporation, Celltrion, Enterome, Ferring, Genentech, Janssen and Janssen, Medimmune, Merck \& Co., Pfizer, Protagonist, Second Genome, Seres, Shire, Takeda, Theradiag, Theravance Biopharma; stock options in Intestinal Biotech Development, Genfit; research Grants from AbbVie, Takeda, Janssen and Janssen. MKVA: none.

\section{Author Contributions}

All authors have made significant contributions to the research described in this manuscript. JB: study concept and design, acquisition of data, analysis and interpretation of data, manuscript writing. RU: study concept and design, interpretation of data, manuscript writing. JFC: study concept and design, interpretation of data, critical revision of the manuscript. MVP, IV, FB, MKAV: acquisition of data, interpretation of data, critical revision of the manuscript. All authors approved the final manuscript as well as the authorship list. JB is the guarantor of the article.

\section{References}

1. Ungaro R, Mehandru S, Allen PB, Peyrin-Biroulet L, Colombel J-F. Ulcerative colitis. Lancet 2017;389:1756-70.

2. Silverberg MS, Satsangi J, Ahmad T, et al. Toward an integrated clinical, molecular and serological classification of inflammatory bowel disease: report of a Working Party of the 2005 Montreal World Congress of Gastroenterology. Can J Gastroenterol 2005;19 Suppl A:5A-36A.

3. Moum B, Ekbom A, Vatn MH, et al. Clinical course during the $1^{\text {st }}$ year after diagnosis in ulcerative colitis and Crohn's disease. Results of a large, prospective population-based study in southeastern Norway, 1990-93. Scand J Gastroenterol 1997;32:1005-12.

4. Burisch J, Pedersen N, Cukovic-Cavka S, et al.; EpiCom Group. Initial disease course and treatment in an inflammatory bowel disease inception cohort in Europe: the ECCO-EpiCom cohort. Inflamm Bowel Dis 2014;20:36-46.

5. Samuel S, Ingle SB, Dhillon S, et al. Cumulative incidence and risk factors for hospitalization and surgery in a population-based cohort of ulcerative colitis. Inflamm Bowel Dis 2013;19:1858-66.

6. Winther KV, Jess T, Langholz E, Munkholm P, Binder V. Survival and causespecific mortality in ulcerative colitis: follow-up of a population-based cohort in Copenhagen County. Gastroenterology 2003;125:1576-82.

7. Vester-Andersen MK, Vind I, Prosberg MV, et al. Hospitalisation, surgical and medical recurrence rates in inflammatory bowel disease 2003-2011-a Danish population-based cohort study. J Crohns Colitis 2014;8:1675-83.

8. Hoie O, Wolters FL, Riis L, et al.; European Collaborative Study Group of Inflammatory Bowel Disease. Low colectomy rates in ulcerative colitis in an unselected European cohort followed for 10 years. Gastroenterology 2007;132:507-15.
9. Beaugerie L, Itzkowitz SH. Cancers complicating inflammatory bowel disease. N Engl J Med 2015;372:1441-52.

10. Langholz E, Munkholm P, Davidsen M, Binder V. Course of ulcerative colitis: analysis of changes in disease activity over years. Gastroenterology 1994;107:3-11.

11. Roda G, Narula N, Pinotti R, et al. Systematic review with meta-analysis: Proximal disease extension in limited ulcerative colitis. Aliment Pharmacol Ther 2017: doi: 10.1111/apt.14063.

12. Magro F, Rodrigues A, Vieira AI, et al. Review of the disease course among adult ulcerative colitis population-based longitudinal cohorts. Inflamm Bowel Dis 2012;18:573-83.

13. Reinisch W, Reinink AR, Higgins PDR. Factors associated with poor outcomes in adults with newly diagnosed ulcerative colitis. Clin Gastroenterol Hepatol 2015;13:635-42.

14. Torres J, Billioud V, Sachar DB, Peyrin-Biroulet L, Colombel JF. Ulcerative colitis as a progressive disease: the forgotten evidence. Inflamm Bowel Dis 2012:1356-63.

15. Vind I, Riis L, Jess T, Knudsen E, et al. Increasing incidences of inflammatory bowel disease and decreasing surgery rates in Copenhagen City and County, 2003-2005: a population-based study from the Danish Crohn colitis database. Am J Gastroenterol 2006;101:1274-82.

16. Vester-Andersen MK, Prosberg MV, Jess T, et al. Disease course and surgery rates in inflammatory bowel disease: a population-based, 7-year follow-up study in the era of immunomodulating therapy. Am J Gastroenterol 2014;109:705-14.

17. Lynge E, Sandegaard JL, Rebolj M. The danish national patient register. Scand J Public Health 2011;39(7 Suppl):30-3.

18. Burisch J. Crohn's disease and ulcerative colitis. occurrence, course and prognosis during the first year of disease in a European population-based inception cohort. Dan Med J 2014;61:B4778.

19. Langholz E. Ulcerative colitis. An epidemiological study based on a regional inception cohort, with special reference to disease course and prognosis. Dan Med Bull 1999;46:400-15.

20. Munkholm P. Crohn's disease-occurrence, course and prognosis. An epidemiologic cohort-study. Dan Med Bull 1997;44:287-302.

21. Langholz E, Munkholm P, Davidsen M, Nielsen OH, Binder V. Changes in extent of ulcerative colitis: a study on the course and prognostic factors. Scand J Gastroenterol 1996;31:260-6.

22. Solberg IC, Lygren I, Jahnsen J, et al.; IBSEN Study Group. Clinical course during the first 10 years of ulcerative colitis: results from a population-based inception cohort (IBSEN Study). Scand J Gastroenterol 2009;44:431-40.

23. Lakatos L, Kiss LS, David G, et al. Incidence, disease phenotype at diagnosis, and early disease course in inflammatory bowel diseases in Western Hungary, 2002-2006. Inflamm Bowel Dis 2011;17:2558-65.

24. Etchevers MJ, Aceituno M, García-Bosch O, et al. Risk factors and characteristics of extent progression in ulcerative colitis. Inflamm Bowel Dis 2009;15:1320-5.

25. Farmer RG, Easley KA, Rankin GB. Clinical patterns, natural history, and progression of ulcerative colitis. a long-term follow-up of 1116 patients. Dig Dis Sci 1993;38:1137-46.

26. Gower-Rousseau C, Dauchet L, Vernier-Massouille G, et al. The natural history of pediatric ulcerative colitis: a population-based cohort study. Am J Gastroenterol 2009;104:2080-8.

27. Meucci G, Vecchi M, Astegiano M, et al. The natural history of ulcerative proctitis: a multicenter, retrospective study. Gruppo di Studio per le Malattie Infiammatorie Intestinali (GSMII). Am J Gastroenterol 2000;95:469-73.

28. Kim B, Park SJ, Hong SP, Kim TI, Kim WH, Cheon JH. Proximal disease extension and related predicting factors in ulcerative proctitis. Scand $J$ Gastroenterol 2014;49:177-83.

29. Chatzicostas C, Roussomoustakaki M, Potamianos S, et al. Factors associated with disease evolution in Greek patients with inflammatory bowel disease. BMC Gastroenterol 2006;6:21.

30. Ayres RC, Gillen CD, Walmsley RS, Allan RN. Progression of ulcerative proctosigmoiditis: incidence and factors influencing progression. Eur J Gastroenterol Hepatol 1996;8:555-8. 